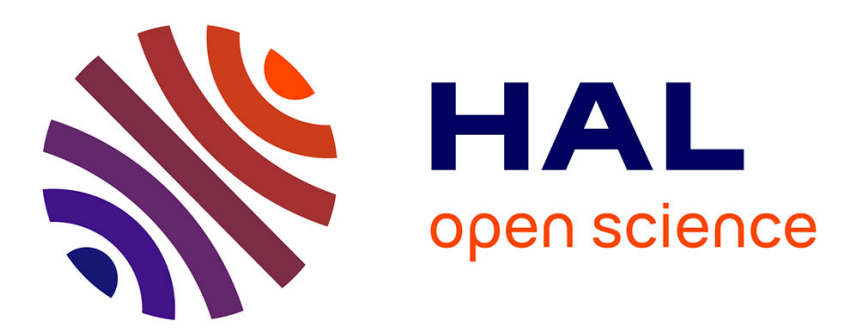

\title{
Mechanical route to the pressure of a fluid adsorbed in a random porous medium
}

\author{
W. Dong
}

\section{To cite this version:}

W. Dong. Mechanical route to the pressure of a fluid adsorbed in a random porous medium. Journal of Chemical Physics, 1995, 102, pp.6570-6573. 10.1063/1.469372 . hal-00006147

\section{HAL Id: hal-00006147 \\ https://hal.science/hal-00006147}

Submitted on 25 May 2021

HAL is a multi-disciplinary open access archive for the deposit and dissemination of scientific research documents, whether they are published or not. The documents may come from teaching and research institutions in France or abroad, or from public or private research centers.
L'archive ouverte pluridisciplinaire HAL, est destinée au dépôt et à la diffusion de documents scientifiques de niveau recherche, publiés ou non, émanant des établissements d'enseignement et de recherche français ou étrangers, des laboratoires publics ou privés. 


\title{
Mechanical route to the pressure of a fluid adsorbed in a random porous medium
}

\author{
W. Dong \\ Institut de Recherches sur la Catalyse, Centre National de la Recherche Scientifique, 2, Avenue Albert \\ Einstein, 69626 Villeurbanne Cedex, France and Laboratoire de Chimie Théorique, Ecole Normale \\ Supérieure de Lyon, 46 Allée d'Italie, 69364 Lyon Cedex 07, France
}

By using different methods, we show how to derive the correct mechanical route to the pressure of a fluid adsorbed in a random porous medium. Some discussions are also made on the thermodynamic consistency of the mechanical route.

\section{INTRODUCTION}

In the last years, the study of porous media has attracted much attention. Various methods of liquid-state theory have been extended successfully to study fluids adsorbed into random porous media. ${ }^{1-11}$ The important work of Madden and Glandt ${ }^{1,2}$ shows that the theoretical description of a fluid adsorbed in a disordered medium can be recast into that of a mixturelike system. This idea has been exploited also in different ways. ${ }^{4-6}$ By using a continuum version of the replica trick, ${ }^{5}$ Given and Stell ${ }^{6}$ have found the exact OrnsteinZernike $(\mathrm{OZ})$ equations and shown that the $\mathrm{OZ}$ equations derived by Madden and Glandt ${ }^{1,2}$ are valid only when Percus-Yevick approximation is used. Several Monte Carlo simulations have been carried out to test approaches borrowed from the familiar liquid-state theories like PercusYevick and hypernetted chain approximations. ${ }^{3,7,8}$ The simulation investigation made by Vega, Kaminsky, and Monson ${ }^{8}$ has a particular importance. They discovered that the virial expression of pressure given by Madden ${ }^{2}$ fails to produce thermodynamically consistent results for the adsorption isotherm calculated from Gibbs-Duhem equation. They proposed also an intuitive approximation for the compressibility which gives very satisfactory results. Subsequently, Ford and Glandt ${ }^{9}$ and Rosinberg et al. ${ }^{10}$ have derived the exact expression of the compressibility by using different methods. Since the compressibility expression is derived directly from a thermodynamic potential, this route assures automatically the thermodynamic consistency. Until now, it is believed that Madden's virial expression of pressure comes from a mechanical definition. The fact that this "virial" route does not lead to thermodynamic consistency provokes a very perplexing and uncomfortable feeling: thermodynamic pressure and mechanical pressure do not coincide. If this were the case, we would get a fundamental trouble, i.e., mechanical equilibrium is not compatible with thermodynamics description. The consequence of this would be also serious in practice. For example, phase equilibria, e.g., liquid-gas coexistence, are determined by thermal, mechanical and chemical equilibrium conditions. A correct determination of phase coexistence requires these equilibrium conditions to be generated from a single thermodynamic potential. Based on the above considerations, we believe that a thermodynamically consistent mechanical route to pressure must exist. Until now, this route has not been found out yet and this issue is addressed in the present work.

\section{RESULTS AND DISCUSSIONS}

We consider the same fluid-matrix system as the one studied by Madden and Glandt. ${ }^{1}$ A rigid host matrix, designated as species 0 , is made by a quench from a thermodynamic equilibrium state. Then, a fluid (species 1) is introduced and allowed to reach equilibrium but the structure of the matrix is not affected by the presence of the latter. Each particular matrix realization is an irregular and highly inhomogeneous system which has a daunting complexity. A statistical mechanics description consists in taking the average over all the matrix realizations. The most important conclusion from the work of Madden and Glandt ${ }^{1}$ is that after the average over matrix realizations is taken, the description of the inhomogeneous fluid-matrix system can be recast into that of a homogeneous mixturelike system. By now, this character has been well exploited. Nevertheless, we will show that this very pleasing aspect should not be overemphasized because the description of some properties, in particular the pressure, is much more akin to that of a "pure" fluid. Now, let us start the main topic of this work, i.e., finding out the mechanical route to the pressure of a fluid adsorbed in a random porous medium.

\section{A. Mechanical equilibrium condition and pressure tensor}

First, we consider a pure mechanics approach. The thermodynamic consistency will be discussed in the next subsection. Let us start from a particular matrix realization. In such a highly inhomogeneous system, the force exerted on a surface element is now a second order tensor. In an external force field, the mechanical equilibrium condition is expressed as

$$
\nabla_{1} \cdot \hat{P}\left(\mathbf{r}_{1} ; \mathbf{q}^{M}\right)=-\hat{\rho}\left(\mathbf{r}_{1} ; \mathbf{q}^{M}\right) \nabla_{1} V\left(\mathbf{r}_{1} ; \mathbf{q}^{M}\right)
$$

where $\hat{P}$ and $\hat{\rho}$ are, respectively, the pressure tensor and the fluid density in the force field, $-\nabla_{1} V\left(\mathbf{r}_{1} ; \mathbf{q}^{M}\right)$, of the matrix and $\mathbf{q}^{M}$ designates explicitly the matrix configuration of a given realization. Any properly defined pressure tensor must satisfy Eq. (1). Otherwise, the mechanical equilibrium cannot be assured. In order to obtain microscopic expressions, we appeal to the microscopic force balance equation, i.e., the first member of the Yvon-Born-Green hierarchy, 


$$
\begin{aligned}
k T \nabla_{1} \hat{\rho}\left(\mathbf{r}_{1} ; \mathbf{q}^{M}\right)= & -\hat{\rho}\left(\mathbf{r}_{1} ; \mathbf{q}^{M}\right) \nabla_{1} V\left(\mathbf{r}_{1} ; \mathbf{q}^{M}\right) \\
& -\int d \mathbf{r}_{2} \nabla_{1} u\left(r_{12}\right) \hat{\boldsymbol{\rho}}^{(2)}\left(\mathbf{r}_{1}, \mathbf{r}_{2} ; \mathbf{q}^{M}\right)
\end{aligned}
$$

where $u\left(r_{12}\right)$ is the pair interaction potential between fluid particles, $\hat{\rho}^{(2)}\left(\mathbf{r}_{1}, \mathbf{r}_{2} ; \mathbf{q}^{M}\right)$ the inhomogeneous two-body distribution function and $k$ and $T$ are respectively the Boltzmann constant and the absolute temperature. By combining Eqs. (1) and (2) and taking the average over matrix realizations denoted as $\langle\cdots\rangle_{M}$, we obtain

$$
\begin{aligned}
\nabla_{1} \cdot\left\langle\hat{\mathrm{P}}\left(\mathbf{r}_{1} ; \mathbf{q}^{M}\right)\right\rangle_{M}= & k T \nabla_{1}\left\langle\hat{\boldsymbol{\rho}}\left(\mathbf{r}_{1} ; \mathbf{q}^{M}\right)\right\rangle_{M}+\int d \mathbf{r}_{2} \nabla_{1} u\left(r_{12}\right) \\
& \times\left\langle\hat{\boldsymbol{\rho}}^{(2)}\left(\mathbf{r}_{1}, \mathbf{r}_{2} ; \mathbf{q}^{M}\right)\right\rangle_{M} .
\end{aligned}
$$

If the disordered porous medium is statistically homogeneous and extends over the whole space, the member on the right hand side (rhs) of Eq. (3) is zero, i.e.,

$$
\nabla_{1} \cdot\left\langle\hat{\mathrm{P}}\left(\mathbf{r}_{1} ; \mathbf{q}^{M}\right)\right\rangle_{M}=0 .
$$

This shows that in this case a scalar pressure can be defined and it is constant throughout. But in this way, we are not able to get any expression for the pressure. However, if the disordered porous sample is not homogeneous over the whole space, the rhs of Eq. (3) is no longer zero. Very recently, Dong, Kierlik, and Rosinberg ${ }^{11}$ have investigated the case in which the porous solid sample occupies only the half space in the region $z>0$. Let us consider again such a system. This will enable us to identify the mechanical fluid pressure inside the porous solid. For this geometry, Eq. (3) can be simplified to

$$
\begin{aligned}
\frac{d p_{\perp}\left(z_{1}\right)}{d z_{1}}= & k T \frac{d \rho\left(z_{1}\right)}{d z_{1}} \\
& +\int d \mathbf{r}_{2} \frac{d u\left(r_{12}\right)}{d z_{1}} \rho_{1}\left(z_{1}\right) \rho_{1}\left(z_{2}\right) g_{11}\left(r_{12}, z_{1}, z_{2}\right)
\end{aligned}
$$

where $p_{\perp}$ is the component of the pressure tensor perpendicular to the surface of porous solid sample, the average over matrix realizations is performed, the subscript " 1 " is introduced to designate fluid species and $g_{11}\left(r_{12}, z_{1}, z_{2}\right)$ is fluid-fluid correlation function. By using the method proposed by Lekner and Henderson ${ }^{12}$ to derive sum rules for liquid-vapor interface, Eq. (5) can be integrated out. For a very clear description of the integration procedure, the interested reader is referred to Ref. 12. The final result after integration is

$$
\begin{aligned}
p(+\infty)-p(-\infty)= & \left\{k T \rho_{1}(+\infty)-\frac{\left[\rho_{1}(+\infty)\right]^{2}}{6}\right. \\
& \left.\times \int d \mathbf{r} r u^{\prime}(r) g_{11}^{+\infty}(r)\right\}-\left\{k T \rho_{1}(-\infty)\right. \\
& \left.-\frac{\left[\rho_{1}(-\infty)\right]^{2}}{6} \int d \mathbf{r} r u^{\prime}(r) g_{11}^{-\infty}(r)\right\} .
\end{aligned}
$$

Since the pressure is isotropic in the region far from the inhomogeneous zone near the solid surface at $z=0$, the subscript " $\perp$ " for the pressure has been dropped. One recognizes readily that the expression in the second curly bracket in Eq. (6) is the pressure of the bulk fluid outside the porous medium, i.e., $p(-\infty)$. Hence, the expression of the pressure of the fluid adsorbed into the porous medium can be identified immediately as

$$
p=k T \rho_{1}-\frac{\rho_{1}^{2}}{6} \int d \mathbf{r} r u^{\prime}(r) g_{11}(r) .
$$

For the simplicity of expression, the arguments and superscript " $+\infty$ " have been dropped. But it should be obviously understood that $g_{11}(r)$ and $\rho_{1}$ in Eq. (7) are respectively the fluid-fluid correlation function and the density of the fluid adsorbed into the porous medium. Here two remarks concerning Eq. (7) are readily in order. First, Eq. (7) is different from the virial expression for pressure given by Madden. ${ }^{2}$ Our result, Eq. (7), does not contain the fluid-matrix cross term in Madden's virial expression. We are going to show shortly that in fact the presence of such a cross term over counts the contribution of the fluid-matrix interaction to the pressure. Second, in their important work, ${ }^{8}$ Vega, Kaminsky and Monson have discovered not only that Madden's virial expression is incorrect but also observed that when the cross term is removed from the Madden's expression, much better thermodynamic consistency can be obtained. Nevertheless, they have neither claimed that the exact expression of the mechanical pressure is in fact Eq. (7) nor looked for a formal proof. We will come back later to comment again the work of Vega et al.. ${ }^{8}$

Now, we will give another way to prove that the correct expression of the mechanical pressure is indeed Eq. (7) and show that the contribution of the fluid-matrix interaction is over counted in Madden's virial expression. In the above derivation of Eq. (7), only the existence of a second order pressure tensor is assumed and then one can proceed without knowing the explicit expression of the pressure tensor. In the following derivation, we will start explicitly from the pressure tensor. In a now classic work of Irving and Kirkwood, ${ }^{13}$ the following expression is derived for the pressure tensor:

$$
\begin{aligned}
\mathrm{P}(\mathbf{r})= & k T \rho(\mathbf{r}) \mathrm{I}-\frac{1}{2} \int d \mathbf{r}_{12} \int_{0}^{1} d \lambda \frac{\mathbf{r}_{12} \mathbf{r}_{12}}{r_{12}} u^{\prime}\left(r_{12}\right) \rho^{(2)}(\mathbf{r} \\
& \left.-\lambda \mathbf{r}_{12}, \mathbf{r}+(1-\lambda) \mathbf{r}_{12}\right)
\end{aligned}
$$

where $I$ is the unit tensor. It is now well known ${ }^{14-16}$ that there is some arbitrariness in the above definition of the pressure tensor. However, the arbitrariness does not affect the result for a homogeneous system. Hence, this arbitrariness is harmless for our present purpose, i.e., finding out the mechanical pressure of a fluid adsorbed into a statistically homogeneous porous medium. It is to be noted that Eq. (8) is valid not only for homogeneous systems but also for inhomogeneous ones. ${ }^{13}$ From Eq. (8), taking the average over matrix realizations for a statistically homogeneous porous medium leads straightforwardly to Eq. (7).

In order to see clearly that there is in fact an over counting of the fluid-matrix interaction in Madden's virial expres- 
sion, it is instructive to note that Madden's expression can be obtained from the following tensor by taking the average over matrix realizations:

$$
\begin{aligned}
\Pi(\mathbf{r})= & \mathrm{P}(\mathbf{r})-\frac{1}{2} \int d \mathbf{r}_{12} \int_{0}^{1} d \lambda \frac{\mathbf{r}_{12} \mathbf{r}_{12}}{r_{12}} v^{\prime}\left(r_{12}\right)\{\rho(\mathbf{r} \\
& \left.-\lambda \mathbf{r}_{12}\right) \sum_{j=1}^{M} \delta\left(\mathbf{q}_{j}-\left[\mathbf{r}+(1-\lambda) \mathbf{r}_{12}\right]\right)+\rho(\mathbf{r}+(1 \\
& \left.\left.-\lambda) \mathbf{r}_{12}\right) \sum_{j=1}^{M} \delta\left(\mathbf{q}_{j}-\left[\mathbf{r}-\lambda \mathbf{r}_{12}\right]\right)\right\}
\end{aligned}
$$

where $\delta(x)$ is the Dirac $\delta$ function and $v^{\prime}\left(r_{12}\right)$ is the derivative of the pair interaction potential between fluid and matrix. Any physically sensible definition of the pressure tensor must satisfy the mechanical equilibrium condition, Eq. (1). With the help of the YBG equation, Eq. (2), it is easy to show that $P(\mathbf{r})$ given in Eq. (8) satisfies the mechanical equilibrium condition but $\Pi(\mathbf{r})$ does not. Hence, it becomes clear that the expression given by Madden ${ }^{2}$ does not have a clear physical significance and the contribution of the fluid-matrix interaction is over counted. In fact, this contribution is already taken into account in the first term on the right hand side of Eq. (8). Here, we would like also emphasize that the form of the equation of state for an ideal gas is not changed even in the presence of the fluid-matrix interaction. Now, we have derived the correct expression of the mechanical pressure by two different methods. In the next subsection, we will make some discussions on the thermodynamic consistency of this mechanical route.

\section{B. Thermodynamic consistency}

The compressibility route to pressure has been found first approximately by Vega et ll. $^{8}$ and then rigorously by Ford and Glandt, ${ }^{9}$ and Rosinberg et al. ${ }^{10}$ It is now established that this route leads to thermodynamically consistent results. ${ }^{8-10}$ We have just found out the rigorous mechanical route to pressure in the last subsection. Now, we will try to examine the thermodynamic consistency of the mechanical route. One possible strategy is to show that Eq. (7) and the compressibility expression given in Refs. 9 and 10 are equivalent. The necessary condition to establish this is that the compressibility route and the mechanical route should be generated from a single thermodynamic potential. Let us consider the grand potential. For an inhomogeneous system corresponding to a given matrix realization, one can obtain the following expression of the grand potential:

$$
\begin{aligned}
\hat{\Omega}\left(\mathbf{q}^{M}\right)= & k T \int d \mathbf{r}_{1} \hat{\rho}\left(\mathbf{r}_{1} ; \mathbf{q}^{M}\right)\left\{\int_{0}^{1} d \alpha \alpha \int d \mathbf{r}_{2} \hat{\boldsymbol{\rho}}\left(\mathbf{r}_{2} ; \mathbf{q}^{M}\right)\right. \\
& \left.\times \hat{c}\left(\mathbf{r}_{1}, \mathbf{r}_{2} ; \mathbf{q}^{M} ;[\alpha \hat{\rho}]\right)-1\right\}
\end{aligned}
$$

by functional integrations along a particular path. ${ }^{17}$ $\hat{c}\left(\mathbf{r}_{1}, \mathbf{r}_{2} ; \mathbf{q}^{M} ;[\alpha \hat{\rho}]\right)$ is the direct correlation function of the in- homogeneous system. When the average over matrix realizations is made for a statistically homogeneous porous medium, we obtain

$$
\begin{aligned}
& -\frac{\left\langle\hat{\Omega}\left(\mathbf{q}^{M}\right)\right\rangle_{M}}{V} \\
& \quad=k T\left[\rho_{1}-\rho_{1}^{2} \int_{0}^{1} d \alpha \alpha \int d \mathbf{r}_{12} c_{11 c}\left(r_{12} ; \alpha \rho_{1}, \rho_{0}\right)\right]
\end{aligned}
$$

where $V$ is the system volume and $c_{11 c}\left(r_{12} ; \alpha \rho_{1}, \rho_{0}\right)$ is the connected part of the direct correlation function between species " 1 " of a mixturelike system at densities $\alpha \rho_{1}$ for species " 1 " (fluid) and $\rho_{0}$ for species "0" (matrix). It should be pointed out that in deriving Eq. (11) from Eq. (10), a nontrivial diagrammatic reduction has to be carried out to establish

$$
\begin{aligned}
& \left\langle\hat{\boldsymbol{\rho}}\left(\mathbf{r}_{1} ; \mathbf{q}^{M}\right) \hat{\boldsymbol{\rho}}\left(\mathbf{r}_{2} ; \mathbf{q}^{M}\right) \hat{c}\left(\mathbf{r}_{1}, \mathbf{r}_{2} ; \mathbf{q}^{M} ;[\alpha \hat{\boldsymbol{\rho}}]\right)\right\rangle_{M} \\
& \quad=\rho_{1}^{2} c_{11 c}\left(r_{12} ; \alpha \rho_{1}, \rho_{0}\right) .
\end{aligned}
$$

The term in $\langle\cdots\rangle_{M}$ on the left hand side of Eq. (12) does not contain any disconnected diagrams. So the averaging over matrix realizations cannot introduce any diagrams belonging to $c_{11 b}(r)$ (the blocking part of the direct correlation function, see Ref. 6 for the precise definitions of $c_{11 c}$ and $c_{11 b}$ ). The left member of Eq. (11) can be identified as the thermodynamic definition of the pressure and the differentiation with respect to $\rho_{1}$ leads straightforwardly to the compressibility expression. Here is another way leading to the compressibility.

Another exact fundamental equation in the density functional theory of inhomogeneous fluids is

$$
-\frac{\delta \hat{\Omega}\left(\mathbf{q}^{M}\right)}{\delta\left(\mu-V\left(\mathbf{r}_{1} ; \mathbf{q}^{M}\right)\right)}=\hat{\rho}\left(\mathbf{r}_{1} ; \mathbf{q}^{M}\right)
$$

where $\mu$ is the chemical potential. It is straightforward to show that $\hat{\rho}\left(\mathbf{r}_{1} ; \mathbf{q}^{M}\right)$ generated from the grand potential through Eq. (13) satisfies the YBG equation, Eq. (2). Then, one can repeat all the derivation from Eq. (1) to Eq. (7). In this way, it can be established that the pressure from the mechanical route, Eq. (7), and the pressure from the compressibility route, Eq. (11) can be generated indeed from the same thermodynamic potential. However, it is not clear whether this is also a sufficient condition for the equivalence of the mechanical route and the compressibility route. The formal proof of this equivalence is still an open problem.

In the recent work of Rosinberg, Tarjus and Stell, ${ }^{10}$ a virial expression for pressure is also given. It is interesting to see if their virial expression agrees with the exact expression of the mechanical pressure, Eq. (7). Because of the complicated form of their expression (see Eq. (46) in Ref. 10), it is very hard to make a comparison in the general case. If the pressure from their virial expression can be identified to the exact mechanical pressure we find in the present work, some miraculous cancelation must occur between some terms in their expression. The work to examine this is under way. ${ }^{18}$

Although the work of Vega, Kaminsky and Monson ${ }^{8}$ strongly suggests that the mechanical pressure given in Eq. (7) is also thermodynamically consistent, there is small but 
appreciable discrepancy between the prediction from mechanical route and their Monte Carlo simulation results (see Fig. 13 in Ref. 8). We believe that the discrepancy is due to numerical inaccuracies, e.g., insufficient statistics for matrix realizations. Therefore, more accurate simulations should be carried out to corroborate our finding. There are also some other possibilities to inquire into the equivalence of the mechanical and the thermodynamic pressures. For examples, virial expansions or very simple models for which analytic solutions exist can be used for this purpose. The work in this direction is being done. ${ }^{18}$

\section{CONCLUSION}

By using different methods, we have found out the correct mechanical route to the pressure of a fluid adsorbed into a random porous medium. It is also shown that the exact mechanical pressure and the compressibility can be generated from the same thermodynamic potential. This and the observation from simulations made by Vega et al. ${ }^{8}$ suggest strongly that the mechanical route found in this work leads also to thermodynamic consistency. However, a formal proof of this remains to be accomplished. The work in this direction is being undertaken.

All the previous works which extend liquid-state theory to random media have revealed and/or well exploited the similarity between a fluid-matrix system and a mixturelike system. A prominent achievement based on this analogy is the derivation of the mixturelike Ornstein-Zernike equations by using the replica method. Nevertheless, this analogy should not lead to the oblivion of the fact that the fluidmatrix system under consideration is intrinsically a highly inhomogeneous system. Unfortunately, there is currently a tendency to overemphasize the similarity to a mixture and to overlook the inhomogeneous aspect. Contrary to what is claimed by Madden and Glandt, ${ }^{1}$ all the thermodynamic quantities cannot be obtained straightforwardly from the usual mixture expression. The expressions for the compressibility and the exact virial pressure are two most striking examples. There is no fluid-matrix cross term in this expres- sions and the presence of the matrix is taken into account only through the fluid-fluid correlation functions. Here, we like to emphasize also that the correlation functions in these pure fluidlike expressions are themselves determined by solving mixturelike Ornstein-Zernike equations. Therefore, there is a kind of duality in the theoretical description of fluid-matrix systems: mixturelikeness and inhomogeneity. Only when this duality is taken into account properly, can a correct description be obtained.

Up to now, no simulation has been carried out in which the direct computation of pressure is made. The result derived in the present work, Eq. (7), lays down the basic principle to perform the direct calculation of the pressure from molecular dynamics simulations. The pressure should be measured by averaging only the virial including fluid-fluid interaction.

\section{ACKNOWLEDGMENT}

The author is indebted to M. L. Rosinberg and E. Kierlik for very stimulating discussions.

${ }^{1}$ W. G. Madden and E. D. Glandt, J. Stat. Phys. 51, 537 (1988).

${ }^{2}$ W. G. Madden, J. Chem. Phys. 96, 5422 (1992).

${ }^{3}$ L. A. Fanti, E. D. Glandt, and W. G. Madden, J. Chem. Phys. 93, 5945 (1990).

${ }^{4}$ D. Chandler, J. Phys.: Condens. Matter 3, F1 (1991).

${ }^{5}$ J. A. Given, Phys. Rev. A 45, 816 (1992).

${ }^{6}$ J. A. Given and G. Stell, J. Chem. Phys. 97, 4573 (1992).

${ }^{7}$ E. Lomba, J. A. Given, G. Stell, J. J. Weis, and D. Levesque, Phys. Rev. E 48, 233 (1993).

${ }^{8}$ C. Vega, R. D. Kaminsky, and P. A. Monson, J. Chem. Phys. 99, 3003 (1993).

${ }^{9}$ D. M. Ford and E. D. Glandt, J. Chem. Phys. 100, 2391 (1994).

${ }^{10}$ M. L. Rosinberg, G. Tarjus, and G. Stell, J. Chem. Phys. 100, 5172 (1994).

${ }^{11}$ W. Dong, E. Kierlik, and M. L. Rosinberg, Phys. Rev. E 50, 4750 (1994).

${ }^{12}$ J. Lekner and J. R. Henderson, Mol. Phys. 39, 1437 (1980).

${ }^{13}$ J. H. Irving and J. G. Kirkwood, J. Chem. Phys. 18, 817 (1950).

${ }^{14}$ A. Harasima, Adv. Chem. Phys. 1, 203 (1958).

${ }^{15} \mathrm{~S}$. Ono and S. Kondo, in Encyclopedia of Physics, edited by S. Flügge (Springer-Verlag, Berlin, 1960) Vol. 10, p. 134.

${ }^{16}$ P. Schofield and J. R. Henderson, Proc. R. Soc. London A 379, 231 (1982).

${ }^{17}$ R. Evans, Adv. Phys. 28, 143 (1979).

${ }^{18}$ M. L. Rosinberg (private communication). 